\title{
DRM-MD approach for bound electron states in semiconductor nano-wires
}

\author{
R. Gospavic ${ }^{1}$, V. Popov ${ }^{1} \&$ G. Todorovic ${ }^{2}$ \\ ${ }^{1}$ Wessex Institute of Technology, Ashurst Lodge, Southampton, UK \\ ${ }^{2}$ Faculty of Civil Engineering, Belgrade, Serbia
}

Abstract

Using the boundary element dual reciprocity method-multi domain (DRM-MD), bound electron states and the corresponding wave functions in semiconductor quantum wires embedded in a matrix were considered. The single circular and rectangular as well as the two near circular quantum wires were analysed. In the case of two coupled quantum wires, the dependence of the resulting wave function and eigenenergies as a function of the distance between wires was calculated. The DRM-MD gave a linear electron state model and the developed numerical approach accurately captured the symmetry breaking and splitting of the degenerated energy states due to the presence of additional wire. According to the symmetry of the structures a suitable mesh reduction was employed and different modes were considered separately. For a case of hetero structures, domain decomposition was used.

Keywords: dual-reciprocity-method, bound states, wave function, quantum wire.

\section{Introduction}

The semiconductor nanostructures due to their unique physical properties have wide range of potential applications in electronic and optoelectronic devices [15]. Latest advances in fabrication technology of semiconductors make it possible to obtain nanostructures in a controllable way and with a wide range of geometries and shapes [6-8], which resulted in a tremendous increase of research interests in nanotechnology and nanostructures in recent years.

As the dimensions of these devices approach nano-scales, quantum effects become significant and quantum mechanical treatment of the problem is 
necessary. Accordingly, there is an increased need for development of efficient numerical models that can be used during the design process.

In the case of quantum wires the free carriers are confined in two directions by potential barriers, forming so called $1 \mathrm{D}$ electron gas $[9,10]$. The lateral dimensions of such structures are much smaller then their length, i.e. they could be considered as wires with infinite length. The electrons are confined to a small region inside the cross section of wires while they can move freely along the wires. If the diameter of wires is getting smaller then the electron states become quantized at discrete energy levels. Such a structures have a number of unique physical characteristics related to their electrical and optical properties $[9,10]$, which make them suitable for various applications.

In this work the DRM-MD approach was employed for the case of constant band effective mass and potential, which produces linear generalized eigenvalue problems and in that sense has advantage relative to the classical BEM which produces a non-linear ones $[12,13]$. An additional advantage of the presented numerical method is that it could be used not only for constant potential and electron effective mass in each region, the case of the classical BEM approach [16], but it can also be used for arbitrary spatial potential distribution and coordinate and energy dependent electron effective masses. In this case a nonlinear eigenvalue system of equations must be solved.

\section{The physical model}

The physical model used to describe the bound states of conductor band electrons inside quantum wires could be expressed by the Schrödinger equation in the one-band effective mass approximation $[11,15]$ :

$$
-\frac{\hbar^{2}}{2} \nabla\left(\frac{1}{m^{*}(x, y, z)} \nabla \Psi(x, y, z)\right)+V(x, y) \cdot \Psi(x, y, z)=E \cdot \Psi(x, y, z)
$$

where $\Psi(x, y, z)$ denotes a wave function belonging to energy level $E, m^{*}(x, y, z)$ is band effective mass which is in general a function of spatial coordinates, $V(x$, $y, z)$ is barrier potential, $\hbar=h / 2 \pi$ and $h$ is Planck's constant. [9, 10, 16].

The z-axis was oriented along the quantum wire axis and it was assumed that the barrier potential depends only on $x$ and $y$ coordinates and that a charge carrier travels freely along z-axis with preset value of the wave number $k_{z}$. It was also assumed that the electron effective mass is constant inside each region and there is a jump in the effective mass only on the junction between different materials. According to these assumptions, the method of variable separation could be used in order to transform the governing equation (1) into a set of equivalent equations as follows: 


$$
\begin{aligned}
& \Psi(x, y, z)=\psi_{T}(x, y) \cdot \psi_{z}(z) \\
& \Delta_{T} \psi_{T}(x, y)-\left(\frac{2 \cdot m^{*}}{\hbar^{2}}(V(x, y)-E)+k_{z}^{2}\right) \cdot \psi_{T}(x, y)=0 \\
& \frac{d \psi_{z}(z)}{d z}+k_{z}^{2} \cdot \psi_{z}(z)=0 \Rightarrow \\
& \psi_{z}(z)=e^{i k_{z} \cdot z} ; \quad \Psi(x, y, z)=\psi_{T}(x, y) \cdot e^{i k_{z} \cdot z}
\end{aligned}
$$

where $\Delta_{T}=\frac{\partial^{2}}{\partial x^{2}}+\frac{\partial^{2}}{\partial y^{2}}, k_{x}=\sqrt{\frac{2 \cdot m^{*}}{\hbar^{2}}}, E=E_{Z}+E_{T}, E_{T}=E-\frac{\hbar^{2} \cdot k_{z}^{2}}{2 \cdot m^{*}}$, and $k_{z}, \psi_{z}, E_{T}, E$ the electron wave number, the wave functions for the free electron in $z$ direction, the electron energy of motion in transversal direction ( $x y$ plane) and the total energy respectively.

At the interface between different regions (contour $\Gamma_{1}$ in figure 1) the next matching conditions exist $[9,10,11,16]$ :

$$
\left.\psi_{1}\right|_{\Gamma_{1}}=\left.\psi_{2}\right|_{\Gamma_{1}},\left.\frac{1}{m_{1}^{*}} \frac{\partial \psi_{1}}{\partial n}\right|_{\Gamma_{1}}=\left.\frac{1}{m_{2}^{*}} \frac{\partial \psi_{1}}{\partial n}\right|_{\Gamma_{1}},
$$

where $m_{1}{ }^{*}$ and $m_{2}{ }^{*}$ denote effective mass in corresponding regions, and $n$ is the normal on the boundary between regions.

Also, on the boundary far from the quantum wires, at distance R, the wave function and its gradient vanish i.e. the following boundary conditions on contour $\Gamma_{2}$ could be used [11]:

$$
\left.\psi\right|_{\Gamma_{2}}=0 ;\left.\quad \frac{\partial \psi}{\partial n}\right|_{\Gamma_{2}}=0
$$

\section{The DRM-MD approach}

The first equation in (2) could be transformed using Green's second identity into the following equation [16]:

$$
\begin{aligned}
& \chi\left(\vec{r}_{1}\right) \cdot \psi\left(\vec{r}_{1}\right)+\oint_{\Gamma_{2}}\left(q^{*}\left(\left|\vec{r}_{1}-\vec{r}_{2}\right|\right) \cdot \psi\left(\vec{r}_{2}\right)-u^{*}\left(\left|\vec{r}_{1}-\vec{r}_{2}\right|\right) \cdot q\left(\vec{r}_{2}\right)\right) \cdot d \Gamma_{1} \\
& =\int_{\Omega} b\left(\vec{r}_{2}\right) \cdot u^{*}\left(\left|\vec{r}_{1}-\vec{r}_{2}\right|\right) \cdot d \Omega \\
& b(x, y)=\left(\frac{2 \cdot m^{*}}{\hbar^{2}}(V(x, y)-E)+k_{z}^{2}\right) \cdot \psi(x, y) ;
\end{aligned}
$$




$$
\begin{aligned}
& q=\frac{\partial \psi}{\partial n} ; \quad q^{*}=\frac{\partial u^{*}}{\partial n} \\
& \chi\left(\vec{r}_{1}\right)=\left\{\begin{array}{ll}
1, & \vec{r}_{1} \in \Omega / \partial \Omega \\
\theta / 2 \pi, & \vec{r}_{1} \in \partial \Omega \\
0, & \vec{r}_{1} \notin \Omega
\end{array} \quad \Gamma=\partial \Omega ; \quad \vec{r}_{1}=\left(x_{1}, y_{1}\right) ; \vec{r}_{2}=\left(x_{2}, y_{2}\right),\right.
\end{aligned}
$$

where $\theta$ is interior angle at boundary point $\vec{r}_{1}$ in radians, $\Omega$ is the problem domain, $\Gamma$ is the boundary contour and $u^{*}$ denotes the fundamental solution for 2D Laplace's operator [17].

The domain integral in (5) could be avoided by using interpolation of the term b over the problem domain $\Omega$ by approximation functions [18].

For the 2D case the most convenient choice for RBF functions are augmented thin plate spline functions (ATPS) [19]. In order to transform the domain integral in (5) into boundary one, an auxiliary function is introduced $[12,13]$.

After spatial discretization of the boundary contour $\Gamma$, using linear elements for interpolating unknown wave function $\psi$ and its normal derivative $q$, boundary integrals could be evaluated and using collocation technique this procedure could be repeated for each boundary as well as interior nodes. In this way a linear generalized eigenvalue problem is obtained. After integral evaluations, (5) can be written in following matrix form [12]:

$$
\begin{aligned}
& H \cdot \psi-G \cdot Q=(H \cdot \hat{U}-G \cdot \hat{Q}) \times F^{-1} \times b ; \\
& \psi=\left[\psi_{1}, \cdots, \psi_{\mathrm{N}_{\psi}}\right] ; \quad Q=\left[\mathrm{q}_{1}, \cdots, \mathrm{q}_{\mathrm{N}_{\mathrm{q}}}\right] \\
& b=\left(\frac{2 \cdot \mathrm{m}^{*}}{\hbar^{2}} \cdot \mathrm{V}+\mathrm{k}_{\mathrm{z}}^{2}-\frac{2 \cdot \mathrm{m}^{*}}{\hbar^{2}} \cdot \mathrm{E}\right) \cdot \psi,
\end{aligned}
$$

where $\psi_{i}$ and $q_{i}$ are unknown nodal values of the wave function and its normal derivate, respectively. Matrix $\boldsymbol{F}$ is obtained from (7) and (8). The above relation corresponds to the case of the constant potential and effective mass in each region separately.

Similar sets of equations are obtained in each of the sub-domains and a system matrix can be assembled. In this way the generalized eigenvalue problem is obtained.

\section{Numerical results}

In all presented numerical result the software ARPACK was used for finding bounded energies and wave functions of the corresponding linear eigenvalue problems. In all examples the field variables and derivatives were represented using linear interpolation over the elements while on the corners and curved contours, linear discontinues elements were used [12]. For the DRM interpolation the ATPS radial basis functions were used. In all numerical examples the considered structures consist of two media and according to the 
physical model given in (1) the electron effective mass has different values in each region.

\subsection{Case study 1}

For the case of a cylindrical wire with constant potentials inside and outside the wire, the bounded energies could be expressed in analytical form [9].

If the potential is a symmetrical function of the coordinates, the wave functions are either even or odd functions of the $x$ and $y$ coordinates and could be classified in four modes, where indexes $i$ and $j$ in the wave function $\psi_{i j}$ indicate the symmetry regarding $x$ and $y$ coordinates respectively $(i, j=S$ for a symmetrical function and $i, j=A$ for asymmetrical one). All these wave functions are orthogonal and thus they are base states, i.e. any bound state could be represented as a linear combination of these four base states.

In Table 1 the numerical results for the bounded energies obtained by the DRM-MD were compared with the analytical ones, in the case of cylindrical wires. The numerical parameters have the following values: $m_{1}{ }^{*}=0.00665 \mathrm{~m}_{\mathrm{e}}$; $m_{2}{ }^{*}=0.00858 \mathrm{~m}_{e} ; V_{0}=320 \mathrm{meV} ; k_{z}=0 \mathrm{~nm} ; r=5 \mathrm{~nm}$ where $m_{e}$ is the free electron mass. These parameters correspond to the $\mathrm{GaAs} / \mathrm{Ga}_{0.63} \mathrm{Al}_{0.37} \mathrm{As}$ structure [11].

Table 1: Comparison of numerical results obtained using the DRM-MD with six sub-domains and analytical solution for the case of cylindrical wires.

\begin{tabular}{|c|c|c|}
\hline mode & DRM-MD [meV] & Analytical [meV] \\
\hline \multirow{2}{*}{ SS } & 75.983 & 76.06 \\
\cline { 2 - 3 } & 317.8111 & 317.2 \\
\hline AA & 317.6100 & 317.2 \\
\hline SA & 188.8149 & 188.5605 \\
\hline AS & 188.8122 & 188.5605 \\
\hline
\end{tabular}

Due to the Hamiltonian's symmetry the quantum states $\psi_{S A}$ and $\psi_{A S}$ are degenerated, i.e. both states have the same energy even though the wave functions are different and each linear combination of these states are also bounded states with same energy and correspond to the rotation of the coordinate system. Also, the $\psi_{A A}$ state and the second states in $S S$ mode $\left(\psi_{S S 2}\right)$ are degenerated and the same explanation holds.

\subsection{Case study 2}

In this case two identical cylindrical wires with constant potential inside and outside the wires were considered. The material parameters, the wave number in the axial direction and the dimensions of the wires were the same as in the pervious case. The calculations were performed for two different distances between wires $(d=4$ and $10 \mathrm{~nm})$ and for SS mode. In table 2 the obtained numerical results are summarized. 
Table 2: $\quad$ Numerical results for bounded energies in SS mode obtained using the DRM-MD for the case of two identical cylindrical wires for different distance $d[\mathrm{~nm}]$ between them (radius of wires $=5 \mathrm{~nm}$, number of sub-domains for each of the cases is denoted with SD).

\begin{tabular}{|c|c|c|}
\hline mode & $\begin{array}{c}\text { energy } \\
{[\mathrm{meV}] ;} \\
\mathrm{d}=4 \mathrm{~nm} ; 5-\mathrm{SD}\end{array}$ & $\begin{array}{c}\text { energy }[\mathrm{meV}] \\
\mathrm{d}=10 \mathrm{~nm} ; 7-\mathrm{SD}\end{array}$ \\
\hline \multirow{2}{*}{$\mathrm{SS}$} & 75.259 & 75.464 \\
\cline { 2 - 3 } & 185.668 & 188.146 \\
\cline { 2 - 3 } & 313.713 & 316.335 \\
\hline
\end{tabular}

The normalized wave functions and contour plots for the first state in SS mode for the case of $d=4 \mathrm{~nm}$, are shown in figures 1 (a)-(b).

\subsection{Case study 3}

In this case study one rectangular wire with dimensions $20 \times 10 \mathrm{~nm}$ was considered. The material parameters were the same as in the previous cases and the potential barrier between different materials was $276 \mathrm{meV}$ and $k_{z}=0 \mathrm{~nm}[11]$.

Domain decomposition with ten sub-domains was used with total number of nodes 1739 of which 798 on the boundary and 941 interior nodes. The wave function and contour plot for the fourth quantum state in $S S$ mode are presented in Figure 2(a)-(b).

\section{Conclusions}

The boundary element DRM-MD approach was developed and applied for calculation of bounded electron states of quantum wires. In order to describe the conduction electrons bound states inside the quantum wires the one-band effective mass approximation was used for solving the Schrödinger equation. The present approach employed linear interpolation of field variables over the boundary elements.

This approach has advantage in respect to the classical BEM approach as it leads to a linear eigenvalue problem compared to the classical BEM which results in a non linear eigenvalue problem. Additional advantage compared to the classical BEM is that the DRM-MD approach can be used not only in the case of constant potential and effective mass inside each region but also for the case when potential and effective mass vary in space [14].

The developed numerical scheme was tested on three case studies: (i) a single cylindrical wire, (ii) two identical cylindrical wires, and (iii) a single rectangular quantum wire. In the case of a single cylindrical wire the results were compared with analytical solution and good agreement was observed. 


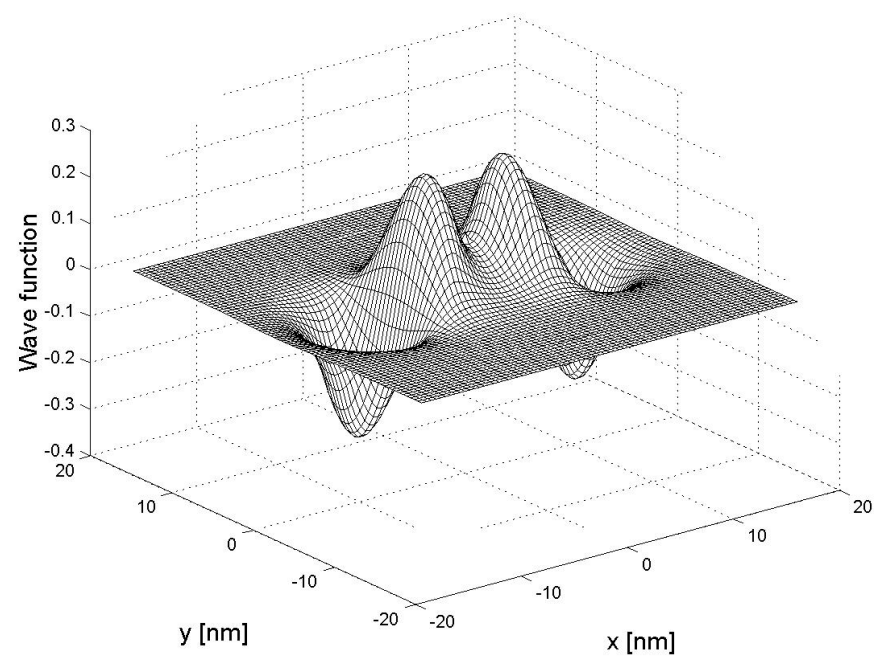

(a)

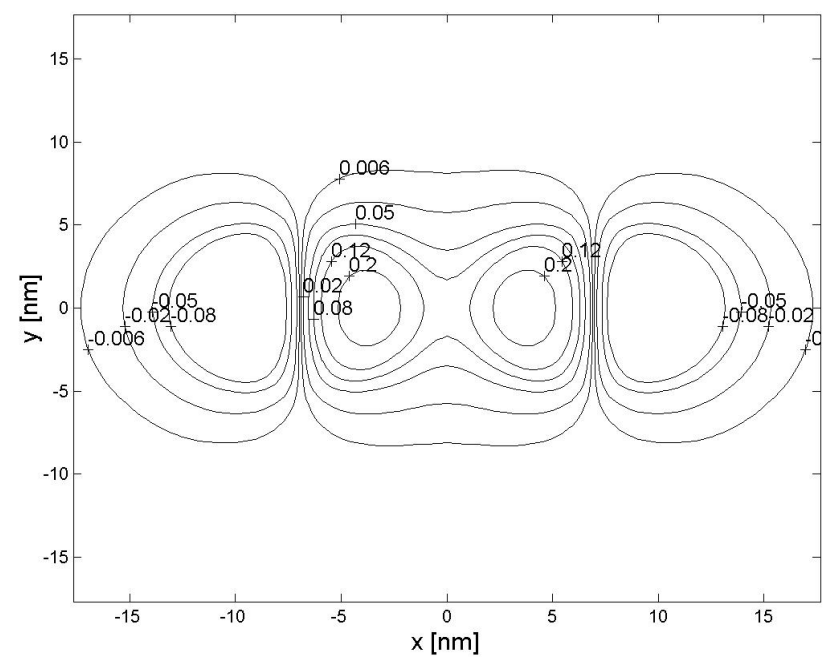

(b)

Figure 1: $\quad$ The normalized wave function and contour plot for the first state in SS mode (table 2) for the case of two identical cylindrical quantum wires (radius of wires $=5 \mathrm{~nm}$, distance between wires $=4 \mathrm{~nm}$, potential barrier $320 \mathrm{meV}$, bound energy $75.259 \mathrm{meV}$ ). 


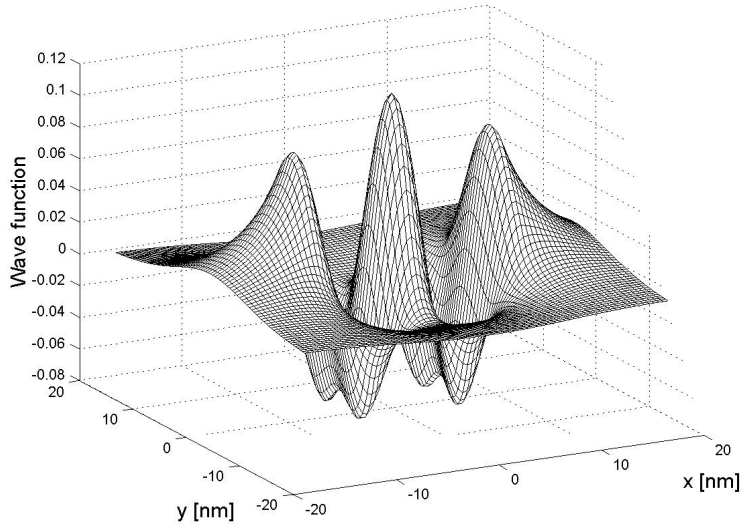

(a)

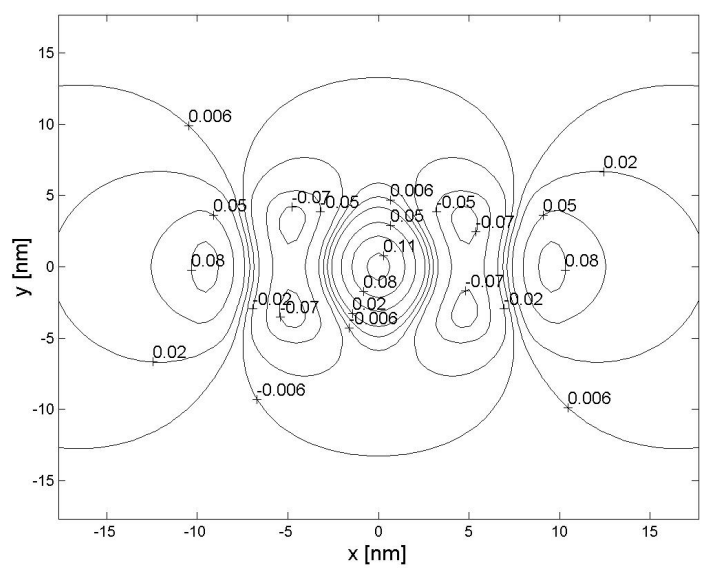

(b)

Figure 2: The normalized wave function and contour plot for the fourth state in SS mode (table 2) for the case of one rectangular quantum wire (dimension $20 \times 10 \mathrm{~nm}$, potential barrier $276 \mathrm{meV}$, bound energy $272.801 \mathrm{meV})$

In the case of the two identical wires two different distances between the wires were considered. In all three cases the domain decomposition technique was used, the shapes and sizes of the subdomains were adjusted according to the physical sub-domains of the considered structure and potential and effective mass were considered to be constant in each sub-domain.

In the case of symmetric potential the problem domain was reduced only to the first quadrant of the rectangular coordinate system leading to high reduction in the mesh used. 
The developed numerical approach could be used for more complex structures such as an arbitrary number of quantum wires arranged in lattice, a case of potential or effective mass varying in space or energy-dependent effective mass.

\section{References}

[1] C. Weisbuch, B. Vinter, Quantum Semiconductor structures: Fundamentals and Applications Academic Press, San Diego, 1991.

[2] P. Havu, M.J. Puska, R.M. Nieminen, Electron transport through quantum wires and point contacts, Physical Review B 70, 233308 (1-4), 2004.

[3] T. Morimoto, Y. Iwase, N. Aoki, T. Sasaki, Y. Ochiai, Nonlocal resonant interaction between coupled quantum wires, Applied Physics Letters $\mathbf{8 2}$ (22), 3952-3954, 2003.

[4] R. Bhat, E. Kapon, S. Simhony, E. Colas, D.M. Hwang, N.G. Stoffel, M.A. Koza, Quantum wire lasers by OMCVD growth on nonplanar substrates, Journal of Crystal Growth 107, 716-723 1991.

[5] J.L. Merz, P.M. Petroff, Making quantum wires and boxes for optoelectronics devices, Materials Science and Engineering B9, 275-284, 1991.

[6] T. Takebe, T. Watanabe, K. Fujita, Fabrication of quantum wires and dots on GaAs (111)A patterned substrates by molecular beam epitaxy, Superlattices and Microstructures 24 (1),1-6 1998.

[7] Ch. Heyn, C. Klein, S. Kramp, S. Beyer, S. Grünther, D. Heitmann, W. Hansen, Fabrication of quantum wires by in-situ ion etching and MBE overgrowth, Journal of Crystal Growth 227-228, 980-984, 2000.

[8] E. Giovine, E. Cianci, V. Foglietti, A. Notargiacomo, F. Evangelisti, Nanofabrication of quantum wires on (100) Si and SiGe by shifted-resist pattern and anisotropic wet etching, Microelectronic Engineering 53, 217 219, 2000.

[9] P. Harrison, Quantum Wells, Wires and Dots (Wiley, New York, 2000).

[10] D.K. Ferry, S.M. Goognick, Transport in Nanostructures (Cambridge University Press, Cambridge, 1997.

[11] E.P. Pokatilov, V.A. Fonoberov, S.N. Balaban, V.M. Fomin, Electron states in rectangular quantum well wires (single wires, finite and infinite lattices), J. Phys.: Condens. Matter 12 (42), 9035-9052, 2000.

[12] V. Popov, H. Power, DRM-MD approach for the numerical solution of gas flow in porous media, with applications to landfill, Eng Anal Bound Elem. 23, 175-188, 1999.

[13] V. Popov, H. Power. The DRM integral equation method: an efficient approach for the numerical solution of domain dominant problems, International Journal for Numerical Methods in Engineering, 44, 327353, 1999.

[14] F. Gelbard, K.J. Malloy, Modeling Quantum Structures with the Boundary Element Method, Journal of Computational Physics, 172, 19-39, 2001. 
[15] D. Indjin, G. Todorovic, V. Milanovic, Z. Ikonic, On numerical solution of the Schrödinger equation: the shooting method revised, Computer Physics Communication, 90, 87-94, 1995

[16] G. Bastarad, Wave mechanics applied to semiconductor heterostructure, Les editions de physique, Les Ulis Cedex, 1988.

[17] C.A. Berbbia, J.C. Tells, L.C. Wrobel, Boundary element techniques, Springer, Berlin, Heidelberg, New York, Tokyo, 1984.

[18] P.W. Patridge, C.A. Brebbia, L. Wrobel, The dual reciprocity boundary element method Computational Mechanics Publication, Southampton and Elsevier Applied Science, London \& New York, 1992.

[19] M.D. Buhmann, Radial basis functions: theory and implementations, University Press, Cambridge, 2003. 